\title{
The Outcomes of Endoanal Ultrasound and Three-Dimensional, High-Resolution Anorectal Manometry do not Predict Fecal Incontinence
}

\author{
Maxime M van Meegdenburg ${ }^{1}$, Hendrik M van Dullemen MD², M Trzpis, PhD ${ }^{1 *}$, Paul MA Broens MD ${ }^{1,3}$ \\ ${ }^{1}$ Department of Surgery, Anorectal Physiology Laboratory, University of Groningen, University Medical Center Groningen, Groningen, the Netherlands \\ ${ }^{2}$ Department of Gastroenterology and Hepatology, University of Groningen, University Medical Center Groningen, Groningen, the Netherlands
}

${ }^{3}$ Department of Surgery, Division of Pediatric Surgery, University of Groningen, University Medical Center Groningen, Groningen, the Netherlands

Submission: March 23, 2021; Published: April 01, 2021

*Corresponding author: M Trzpis, Anorectal Physiology Laboratory, University Medical Center Groningen, PO Box 30001, 9700 RB Groningen, Netherlands

\begin{abstract}
Endoanal ultrasound (EUS) is the gold standard for diagnosing anal sphincter defects often seen in patients with fecal incontinence (FI). Threedimensional, high-resolution anorectal manometry (3D-HRARM) is a newer technique that might also be used to diagnose sphincter defects. We aimed to investigate whether FI is associated with anal sphincter defects detected by EUS and 3D-HRARM. Retrospectively, we included all adult patients who had undergone EUS and 3D-HRARM for FI, between January 2012 and February 2015 (N = 37). During 3D-HRARM, the presence of sphincter defects was examined in rest and during maximal anal sphincter contraction. All patients also underwent a balloon retention test to objectively determine whether they suffered from FI for solid stool. Of the 37 patients, 12 patients (32\%) suffered from FI. The presence of a sphincter defect detected with EUS, and with 3D-HRARM during contraction, was not associated with the prevalence of FI and no significant correlations were found between these variables. The presence of a sphincter defect, detected by 3D-HRARM in rest, was negatively correlated with the presence of FI $\left(\mathrm{r}_{\mathrm{s}}-.372, P=.024\right)$. Moreover, the prevalence of sphincter defects was lower in patients with FI, detected by 3D-HRARM in rest, than in patients without FI ( $13 \%$ versus $88 \%, P=.035)$. FI is not associated with anal sphincter defects detected by EUS and 3D-HRARM. The outcomes of EUS and 3D-HRARM do not thus predict the presence of FI. Instead, extensive anorectal function tests should be performed to form a complete picture of a patient's anorectal functions and to determine the underlying causes of FI.
\end{abstract}

Keywords: Anal canal; Endosonography; Fecal incontinence; Manometry

\section{Introduction}

Fecal incontinence (FI) is a frequent, multifactorial disease that can lead to a decreased quality of life and has a significant economic impact [1]. Several patients with FI suffer from anal sphincter defects that are often caused by obstetrical trauma or anorectal surgery [1-4]. Currently, the gold standard for diagnosing anal sphincter defects is endoanal ultrasound (EUS), a simple, well-tolerated, reproducible, and inexpensive technique [5-7]. Experienced EUS practitioners reach a sensitivity of 95\% to $100 \%$ and a specificity of $75 \%$ to $100 \%$ for diagnosing anal sphincter defects $[8,9]$. Based on the EUS results some patients undergo anal sphincter repair with the aim of improving fecal continence. A systematic review, however, showed that after anal sphincter repair the long-term effect on FI can be disappointing [10].
Three-dimensional, high-resolution anorectal manometry (3D-HRARM) was introduced recently. The catheters used in 3D-HRARM are based on a new technology that provides better spatial resolution of the sphincter pressure profile compared to the catheters used previously. This technique can therefore be used to evaluate anal sphincter function and to diagnose anal sphincter defects. Vitton \& colleagues [11] demonstrated that the results of 3D-HRARM correlate with the results of EUS, but the level of agreement was not sufficient to conclude that 3D-HRARM can be reliably used to diagnose anal sphincter defects. They did, however, fail to relate their findings to the presence of FI. Rezaie \& colleagues [12] showed that 3D-HRARM has a negative predictive value of $92 \%$ for sphincter defects and might therefore be used in patients with a low suspicion of a sphincter defect to rule out the 
presence of a sphincter defect. Moreover, they found comparable Cleveland Clinic Fecal Incontinence scores between patients with or without a sphincter defect diagnosed with EUS. Likewise, Thomas and colleagues did not find a significant difference in Cleveland Clinic FI scores between patients with and without a sphincter defect detected by EUS [13]. Our aim was to investigate whether an association exists between anal sphincter defects detected by EUS and 3D-HRARM, and FI. This approach will enable us to evaluate whether EUS or 3D-HRARM can predict FI.

\section{Materials and Methods}

\section{Patients}

Retrospectively, we reviewed the medical records of patients older than 17 who had undergone 3D-HRARM and EUS on account of chronic FI between January 2012 and February 2015 ( $\mathrm{N}=37$ ). Directly after referral the patients who were originally referred for 3D-HRARM were also signed-up for EUS and vice versa. We included all 37 patients in the analysis. Anorectal manometry and EUS were performed on different days, in no order, and without bowel preparation. This study was conducted at the Anorectal Physiology Laboratory of University Medical Center Groningen, the Netherlands, in compliance with the requirements of our local medical ethics review board.

\section{Anorectal manometry}

We performed 3D-HRARM and the balloon retention test. The patients were all tested by a single experienced operator. During the 3D-HRARM procedure the patients lay in the left lateral recumbent position, while during the balloon retention test, they sat upright on a commode to mimic the natural situation.

\section{Measuring equipment}

Data were recorded and analyzed with solar, gastrointestinal high-resolution manometry equipment, Version 8.23 (Medical Measurement Systems, Enschede, the Netherlands). Different catheters were used for 3D-HRARM and the balloon retention test.

\section{Three-dimensional high resolution anorectal manometry}

For this test we used a HRAM-3D-catheter with an outer diameter of $12 \mathrm{~F}$ that measures circumferential pressure every 8 $\mathrm{mm}$ over a total length of $3.2 \mathrm{~cm}$. The catheter was inserted into the anal canal. We measured anal basal pressure as soon as the patients were completely at ease. The patients were then asked to maximally squeeze their anal sphincters, which enabled us to measure maximal anal pressure. An anal sphincter defect in rest was defined as a decrease in anal basal pressure of minimal $20 \%$ over the total length of the anal sphincter. An anal sphincter defect during contraction was defined as a decrease in maximal anal pressure of minimal $20 \%$ over the total length of the anal sphincter.

\section{Balloon retention test}

We used a Unisensor K12981, solid-state (Boston type) circumferential catheter with an outer diameter of $12 \mathrm{~F}$ to measure circumferential pressure every $8 \mathrm{~mm}$ over a total length of $6.8 \mathrm{~cm}$ into the rectum (Catheter A). We used a Unisensor K14204 catheter with an outer diameter of $14 \mathrm{~F}$ with two microtip sensors to connect the rectal balloon, to inflate it, and to register the pressure inside the balloon (Catheter B). The solar, gastrointestinal, highresolution manometry equipment corrected the pressure inside the balloon for the resistance of the balloon itself, so that only the real pressure in the rectum was recorded.

We inserted Catheter A into the anal canal and Catheter B into the rectum. Catheter A was fixed onto the buttocks near the anal canal with adhesive tape to prevent it from slipping during the procedure. As soon as the patient was completely at ease, we filled the balloon with water of $37^{\circ} \mathrm{C}(1.0 \mathrm{~mL} /$ second $)$. The patient was asked to retain the balloon for as long as possible. Patients, who lost the balloon before reaching maximum tolerable volume, were classified as fecally incontinent for solid stool.

\section{Endoanal ultrasound}

A single experienced operator performed the EUS using a Hitachi processor with a rigid, $360^{\circ}$ viewing rectal probe with a frequency of $7 \mathrm{MHz}$ (Hitachi Medical Systems, Japan). We performed the examination with the patient lying in the left lateral position. We lubricated the tip of the probe with ultrasound jelly and then covered it with a condom that we had also lubricated to facilitate insertion of the probe. We subsequently inserted into the anus to obtain the images. Internal and external anal sphincter thickness was measured at four quadrants $(12,3,6$, and 9 o'clock). We defined an internal anal sphincter defect as an echogenic interruption and an external anal sphincter defect as a hypoechogenic interruption $[11,14]$. We documented and photographed the anal sphincter defects in the most proximal and distal part of the anal sphincter.

\section{Statistical analysis}

The data was analyzed with IBM SPSS Statistics for Windows, Version 23.0 (IBM Corp, Armonk, NY, USA). Because of the nonnormal distribution of the data, we reported median, minimum, and maximum values. Spearman correlations were used to compare the results of EUS and anorectal manometry. We used univariate logistic analyses to determine the influence of a sphincter defect on FI. Statistical significance was defined as $P \leq$ .05 .

\section{Results}

Thirty four out of the 37 patients (92\%) were women with a median age of 62 years, ranging from 26 to 76 years. The median time between EUS and anorectal manometry was two months, 
ranging from 0 to 7 months). During the balloon retention test 12 patients $(32 \%)$ lost the rectal balloon before reaching maximum

tolerable volume and were therefore diagnosed as fecally incontinent for solid stool (Table 1).

Table 1: Patient characteristics and their fecal continence for solid stool as measured with the balloon retention test.

\begin{tabular}{|l|c|c|}
\hline \multirow{3}{*}{ Patient Characteristics } & Number of patients & 37 \\
\cline { 2 - 3 } & Women & $34(92 \%)$ \\
\cline { 2 - 3 } & Age (years) & $62(26-76)$ \\
\hline \multirow{2}{*}{ Balloon Retention Test } & Patients incontinent for solid stool & $12(32 \%)$ \\
\cline { 2 - 3 } & Volume at which rectal balloon was lost $(\mathrm{mL})$ & $145(45-500)$ \\
\hline
\end{tabular}

Data are presented as number (\%) or median (range).

\section{The prevalence of sphincter defects detected by EUS and 3D-HRARM in relation to FI}
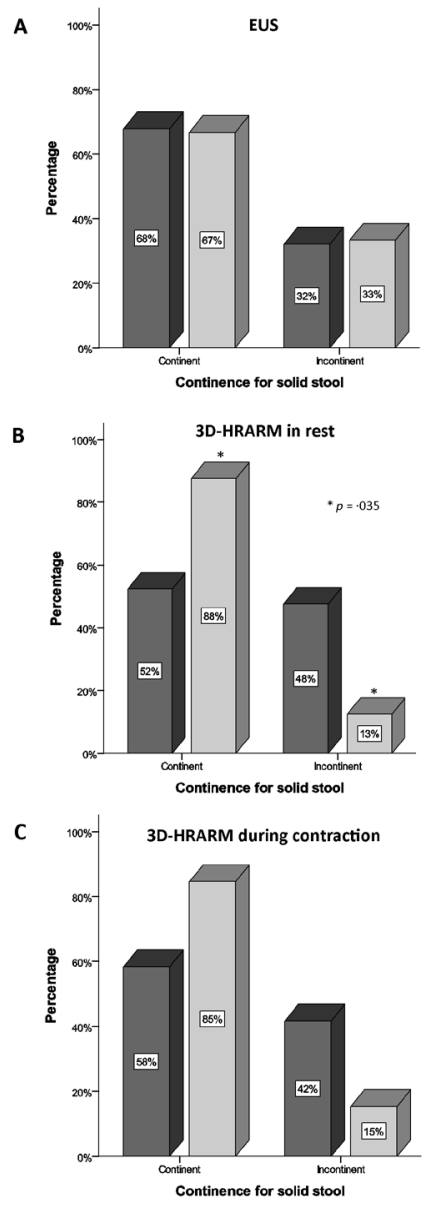

Figure 1: The presence of anal sphincter defects in patients with and without fecal incontinence (FI) according to endoanal ultrasound (EUS) (A) and three-dimensional high-resolution anorectal manometry (3D-HRARM) at rest (B) or during contraction of the anal sphincter $(C)$

A. Patients with an anal sphincter defect detected by EUS did not suffer from FI more often than patients without an anal sphincter defect detected by EUS.

B. Patients with a functional anal sphincter defect at rest, detected by 3D-HRARM, did not suffer from FI more often than patients without a functional anal sphincter defect detected by 3D-HRARM at rest.

C. Patients with a functional anal sphincter defect during contraction, detected by 3D-HRARM, were significantly less often fecally incontinent than patients without a functional anal sphincter defect during contraction detected by 3D-HRARM. 
The prevalence of FI did not differ significantly between patients with and without a sphincter defect detected by EUS (33\% versus $32 \%$, Figure $1 \mathrm{~A}$ ) or 3D-HRARM during contraction (15\% versus $42 \%$, Figure $1 \mathrm{~B}$ ). These findings were in line with correlation analyses that showed no significant correlations between FI and the presence of a sphincter defect detected by EUS, or by 3D-HRARM during contraction (Table 2).

Table 2: Spearman correlations between the outcomes of EUS and 3D-HRARM, regarding the presence of sphincter defects, and their measured incontinence for solid stool.

\begin{tabular}{|c|c|c|c|c|c|}
\hline & & 1 & 2 & 3 & 4 \\
\hline 1 & Balloon retention test MTV or MRV = continence solid stool & 1 & & & \\
\hline 2 & Sphincter defect 3D-ARM in rest (yes/no) & $-0.372^{*}$ & 1 & & \\
\hline 3 & Sphincter defect 3D-ARM during contraction (yes/no) & -0.268 & $0.615^{\wedge}$ & 1 & \\
\hline 4 & Sphincter defect EUS (yes/no) & 0.011 & 0.014 & 0.111 & 1 \\
\hline
\end{tabular}

${ }^{*} p=.024, \wedge p<.001$

Surprisingly, of the patients with a sphincter defect detected by 3D-HRARM in rest, only $13 \%$ were fecally incontinent, while $88 \%$ were fecally continent ( $P=.035$, Figure $1 C$ ). Furthermore, we found a significantly negative correlation between the presence of a sphincter defect obtained with 3D-HRARM during contraction and FI $\left(\mathrm{r}_{\mathrm{s}}-.372, P=.024\right.$, Table 2$)$. Thus, patients without a sphincter defect are more often fecally incontinent than patients with a sphincter defect.
We also performed univariate logistic regression analyses to predict the influence of a sphincter defect on the presence of FI for solid stool. A sphincter defect detected by EUS, or by 3D-HRARM during contraction, did not significantly predict the presence of FI $(P=.947$ and $P=.117$, respectively). Patients with a sphincter defect detected by 3D-HRARM in rest, however, had a significantly decreased risk of FI compared to patients without a sphincter defect according to 3D-HRARM during contraction (OR .157, 95\% CI, .028-.870, $P=.034$ ).

\section{Association between the presence of sphincter defects according to EUS and 3D-HRARM}

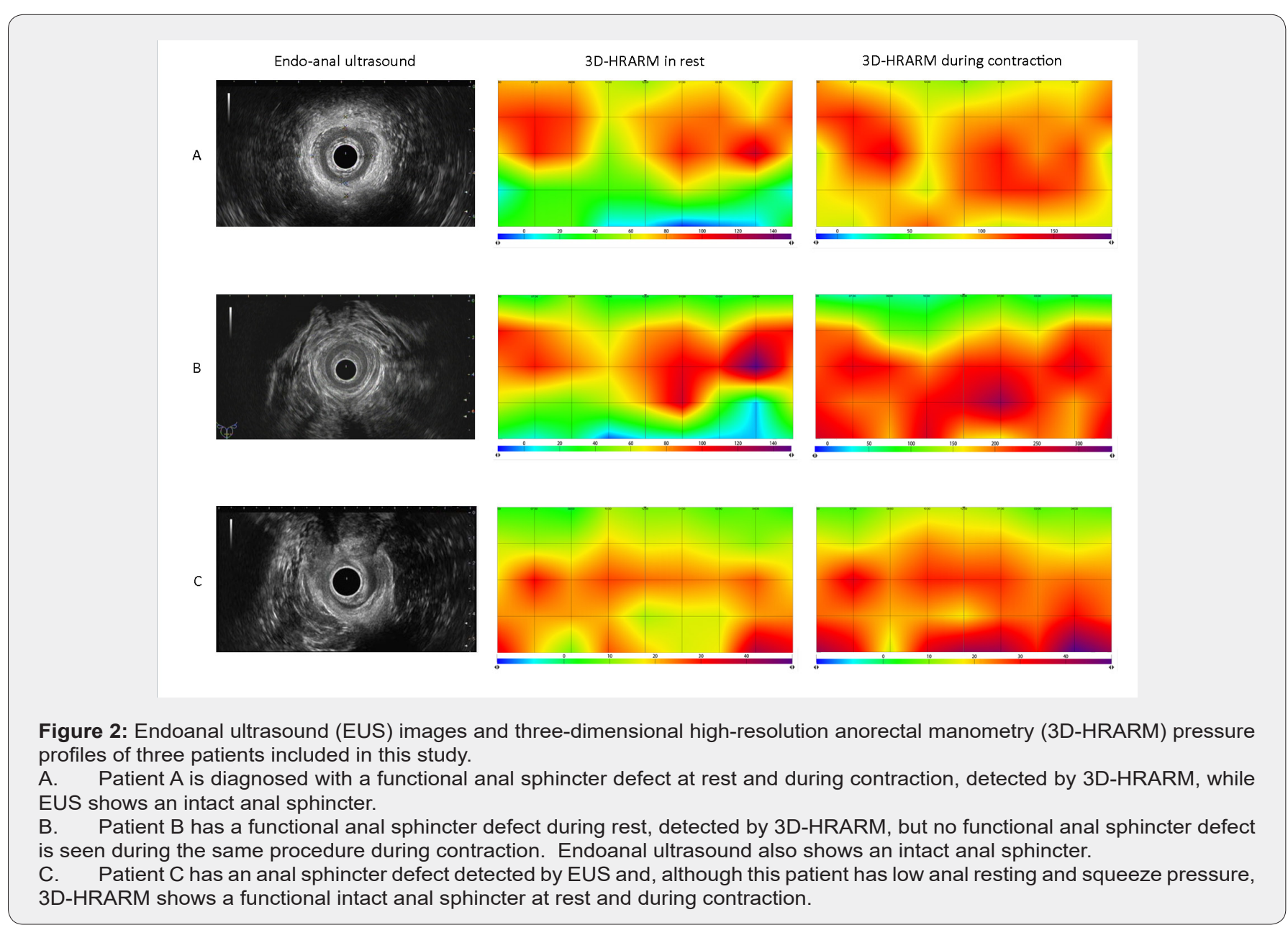


Finally, we compared the results of EUS and 3D-HRARM regarding the presence of a sphincter defect. No significant correlations were found between the detection of a sphincter defect with EUS or 3D-HRARM (Table 2). Furthermore, in 19 patients (51\%) the results of EUS and 3D-HRARM were different regarding the presence of a sphincter defect (Table 3). This finding is also demonstrated in Figure 2 that shows the sphincter condition of three patients included in this study who had undergone both EUS and 3D-HRARM.

Table 3: Presence of sphincter defects according to EUS and 3D-HRARM.

\begin{tabular}{|c|c|c|c|c|}
\hline SD EUS & SD 3D-HRARM in rest & SD 3D-HRARM during contraction & SD n (\%) & Total SD n (\%) \\
\hline- & - & - & $15(41)$ & \multirow{2}{*}{$18(49)$} \\
\hline+ & + & + & $3(8)$ & $8(22)$ \\
\hline+ & + & + & $4(10)$ & \multirow{2}{*}{$19(51)$} \\
\hline- & - & - & $4(10)$ & $1(3)$ \\
\hline+ & + & + & $1(3)$ & \\
\hline+ & - & + & $1(3)$ & \\
\hline
\end{tabular}

\section{Discussion}

To treat FI effectively it is important to perform diagnostic tests to determine its underlying cause. Currently, EUS and 3D-HRARM are the most used diagnostic tools to diagnose sphincter defects, one of the possible underlying causes of FI. Even though EUS can visualize damage to the anal sphincter, it does not measure anal pressure and can therefore not be used to determine anal sphincter functionality. Three-dimensional, HRARM can be used to determine whether and to what extent a sphincter defect has led to a decrease in resting pressure or maximum anal sphincter pressure. This provided additional information about the influence of a sphincter defect on anal sphincter functionality, and thus the possible contribution of a sphincter defect to FI. Our results, however, demonstrated that performing either EUS or 3D-HRARM was insufficient to determine the underlying cause of FI.

We demonstrated that the presence of a sphincter defect detected by EUS, or by 3D-HRARM during contraction, was not associated with FI. When we compared the presence of a sphincter defect detected by 3D-HRARM in rest, we even found a statistically significant negative association with the presence of FI. In contrast to the current dogma that sphincter defects are strongly related to FI, as many as $88 \%$ of the patients with a sphincter defect in rest detected by 3D-HRARM, were fecally continent for solid stool. Thus, the presence of a sphincter defect does not necessarily lead to FI.

Our findings are supported by recent studies by other researchers who did not find any differences in Cleveland Clinic FI scores between patients with or without a sphincter defect diagnosed with EUS [12,13]. Furthermore, several patients underwent anal sphincter repair based on the results of EUS. A recent systematic review, however, showed that three and a half years after anal sphincter repair, $52 \%$ of patients still suffered from FI [10]. While this disappointing result might be explained by the fact that FI is a multifactorial disease, it might also be explained by our finding that anal sphincter defects have less influence on FI than previously thought. Anal sphincter repair should therefore only be considered after extensive anorectal manometry in patients with no other explanations for the FI than an anal sphincter defect.

Only in 18 patients (49\%) did the outcomes of EUS and 3D-HRARM lead to the same diagnosis regarding the presence of a sphincter defect. EUS, however, provides a static image that determines whether there is sphincter damage, while 3D-HRARM is a dynamic test that measures sphincter function. Our result is slightly lower than the results of two other studies that compared the outcomes of EUS with the results of 3D-HRARM in rest. Rezaie \& colleagues [12] found the same diagnosis in 59\% of patients. Vitton \& colleagues [11] showed that the level of agreement between 3D-HRARM and EUS for diagnosing anal sphincter defects was $55 \%$ to $60 \%$. This discrepancy between the results of EUS and 3D-HRARM might be caused by the differences in measuring technique.

During our 3D-HRARM measurements we observed that there were patients in whom a sphincter defect was visible during rest but not during contraction and vice versa. Therefore, we used the results of 3D-HRARM in both rest and during contraction. In the present study, $13 \%$ of the patients fulfilled the criteria for a sphincter defect in rest, but not during contraction, and $6 \%$ had a sphincter defect during contraction, but not in rest. These differences might be explained by the locality of the sphincter defect. Anal sphincter defects that only affect the internal anal sphincter might lead to a sphincter defect in rest but not during contraction, while sphincter defects that only affect the external anal sphincter might lead to a sphincter defect during contraction but not in rest. Nevertheless, we found no significant, positive, correlation between FI and the presence of a sphincter defect diagnosed with 3D-HRARM, during rest or contraction. 


\section{Limitations}

It is a retrospective study consisting of a relatively small cohort of patients.

\section{Conclusion}

The presence of anal sphincter defects detected by either EUS or 3D-HRARM does not seem a suitable predictor of FI for solid stool. For this reason, using these techniques to determine the underlying cause of FI is unreliable and should therefore not be used for this purpose. Consequently, the treatment of FI should not be based solely on the results of these tests. Instead, we recommend performing extensive diagnostic anorectal function tests. By combining the results on these tests, a complete picture can be formed of a patient's anorectal functions. The underlying cause or causes of FI will become clearer, which in turn results in better treatment.

\section{Acknowledgment}

The authors thank Mss. O.J. Pras and T. de Groot for their substantial assistance in the Anorectal Physiology Laboratory and T. van Wulfften Palthe, PhD, for correcting the English manuscript.

\section{References}

1. R D Madoff, S C Parker, M G Varma, A C Lowry (2004) Faecal incontinence in adults. Lancet 364: 621-632.

2. W M Sun, T C Donnelly, N W Read (1992) Utility of a combined test of anorectal manometry, electromyography, and sensation in determining the mechanism of 'idiopathic' faecal incontinence. Gut 33: 807-813.

3. A E Bharucha, J G Fletcher, C M Harper, D Hough, J R Daube, et al. (2005) Relationship between symptoms and disordered continence mechanisms in women with idiopathic faecal incontinence. Gut 54 546-555.

4. A H Sultan, M A Kamm, C N Hudson, J M Thomas, C I Bartram (1993) Anal-sphincter disruption during vaginal delivery. N Engl J Med 329: 1905-1911.
5. P J Law, M A Kamm, C I Bartram (1991) Anal endosonography in the investigation of faecal incontinence. Br J Surg 78: 312-314.

6. S J Burnett, C T Speakman, M A Kamm, C I Bartram (1991) Confirmation of endosonographic detection of external anal sphincter defects by simultaneous electromyographic mapping. Br J Surg 78: 448-450.

7. J J Tjandra, S L Dykes, R R Kumar, C N Ellis, S G Gregorcyk, et al. (2007) Standards Practice Task Force of The American Society of Colon and Rectal Surgeons, Practice parameters for the treatment of fecal incontinence. Dis Colon Rectum 50: 1497-1507.

8. K Hill, S Fanning, M B Fennerty, D O Faigel (2006) Endoanal ultrasound compared to anorectal manometry for the evaluation of fecal incontinence: a study of the effect these tests have on clinical outcome. Dig Dis Sci 51: 235-240.

9. D Saranovic, G Barisic, Z Krivokapic, D Masulovic, A Djuric Stefanovic (2007) Endoanal ultrasound evaluation of anorectal diseases and disorders: technique, indications, results and limitations. Eur J Radiol 61: 480-489.

10. S C Glasgow, A C Lowry (2012) Long-term outcomes of anal sphincter repair for fecal incontinence: a systematic review. Dis Colon Rectum 55: 482-490.

11. V Vitton, W Ben Hadj Amor, K Baumstarck, M Behr, M Bouvier, et al. (2013) Comparison of three-dimensional high-resolution manometry and endoanal ultrasound in the diagnosis of anal sphincter defects. Colorectal Dis 15: e607-11.

12. A Rezaie, S Iriana, M Pimentel, Z Murrell, P Fleshner, et al. (2016) Can 3D high resolution anorectal manometry detect anal sphincter defects in patients with faecal incontinence? Colorectal Dis 19(5): 468-475.

13. G P Thomas, L E Gould, F Casunuran, D A Kumar (2017) A retrospective review of 1495 patients with obstetric anal sphincter injuries referred for assessment of function and endoanal ultrasonography. Int Colorectal Dis 32(9): 1321-1325.

14. M Barthet, P Bellon, E Abou, F Portier, S Berdah, et al. (2002) Anal endosonography for assessment of anal incontinence with a linear probe: relationships with clinical and manometric features. Int J Colorectal Dis 17: 123-128.

\section{Your next submission with JuniperPublishers will reach you the below assets}

- Quality Editorial service

- Swift Peer Review

- Reprints availability

- E-prints Service

- Manuscript Podcast for convenient understanding

- Global attainment for your research

- Manuscript accessibility in different formats

( Pdf, E-pub, Full Text, audio)

- Unceasing customer service

Track the below URL for one-step submission https://juniperpublishers.com/online-submission.php 